\title{
Equine Therapy in the Treatment of Female Eating Disorder
}

\author{
Edward J. Cumella ${ }^{1 *}$, Carrie Boyd Lutter ${ }^{2}$, Alexa Smith Osborne ${ }^{3}$, Zina Kally ${ }^{4}$ \\ ${ }^{1}$ Department of Graduate Psychology, Kaplan University, 6301 Kaplan University Avenue, Fort Lauderdale, FL 33309, USA \\ ${ }^{2}$ Private Practice, Iowa City, IA \\ ${ }^{3}$ School of Social Work, University of Texas at Arlington \\ ${ }^{4}$ Department of Research and Evaluation, Partners in Care Foundatio \\ *Corresponding author: ecumella@kaplan.edu
}

\begin{abstract}
:
The article describes the preconditions and develops the hypothesis concerning the Equine therapy (EQT) is commonly used in the treatment of eating disorder (ED) inpatients, yet studies of its efficacy are lacking. This treatment outcome study therefore compares the efficacy of a standardized inpatient treatment protocol to standard treatment plus EQT among ED subjects. The sample include 72 women ED inpatients between ages 18 and 49, diagnosed with anorexia and bulimia nervosa and eating disorder not otherwise specified. We assesse specific ED and common co-occurring symptoms hypothesized in the literature as amenable to treatment with EQT, including drive for thinness, impaired self-efficacy, interpersonal distrust, impulse dysregulation, depressed mood, and anxiety. Multiple regression analyses controlling for initial symptom severity and quantity of standardized treatment received find that the amount of time patients engaged in EQT in addition to standardized treatment produces statistically significant and clinically meaningful reductions in the six ED symptoms measured. We summarize hypotheses regarding the therapeutic mechanisms that may account for these significant effects of EQT. The incremental value of EQT in addition to standardized inpatient ED treatment in reducing critical ED symptoms may warrant additional investigation into EQT with this patient population.
\end{abstract}

Keywords:

Eating Disorders, Equine Therapy, Hippotherapy

\section{Introduction}

Preliminary research evidence in the form of observational and uncontrolled pilot studies suggests possible efficacy for animal-assisted therapies in treating patients with a range of psychiatric disorders and issues. The disorders for which efficacy has been suggested include depression, childhood internalizing behaviors, and low self-esteem [1-5]; anxiety [6-8]; grief and loss [9]; attention- deficit/hyperactivity disorder [10]; conduct and antisocial personality disorders and childhood externalizing behaviors [5, 11, 12]; dissociative disorders [13]; post-traumatic stress disorder [14-16]; Alzheimers disease and dementia [17-20]; pervasive developmental disorders and learning disabilities [21-25]; chronic mental illnesses [26-31]; and general psychological distress among residential treatment center patients [32, 33] conducted a comprehensive meta-analysis of studies involving animal-assisted therapy. They concluded 
that animal-assisted therapy was associated with moderate effect sizes in improving outcomes in four areas: autism-spectrum symptoms, medical difficulties, behavioral problems, and emotional well-being. They proposed that animal-assisted therapy shows promise as an additive to established interventions and future research should investigate the conditions under which animal-assisted therapy can be most helpful. Nevertheless, of the some 250 studies identified by [33] for their meta-analysis, less than $10 \%$ involved EQT; most considered other forms of animal-assisted therapy, principally canine therapy. Furthermore, only one study compared EQT with standard treatments [5], leaving the question of EQTs therapeutic efficacy largely unanswered. As such, EQT can be said to be in stage 1 of the National Institute of Mental Health model of treatment development [34]. EQT has been repeatedly refined by trials with patients, and pilot tested using simple pre-post designs without a control condition. EQT interventions have been standardized and manualized, with therapist training and certification programs established by the Equine Assisted Growth and Learning Association [35].

Despite the lack of systematic research into its efficacy, inpatient and residential ED treatment facilities often incorporate EQT into their programs. Based on the authors recent survey of all inpatient and residential ED treatment facilities in the United States listed in the Grze 2011 Eating Disorders Catalogue [? ], a comprehensive industry source, 13 of 56, or $24 \%$, of ED programs utilize some form of EQT with their patients. The frequent application of EQT in treating ED patients rests on observational findings among its practitioners [36] which suggest that EQT may yield a variety of psychotherapeutic benefits for ED patients, including enhanced self-confidence and self-efficacy; improved self-concept and self-acceptance; improved awareness of body size; improved interpersonal communication, trust, and decreased social isolation; anxiety reduction; improved mood; better impulse control and assertiveness; and a growth in spiritual wellness and creativity [36-40].

In keeping with EQTs frequent use among ED patients, ED specialists who practice EQT are visible and active members of the Equine Assisted Growth and Learning Association (EAGALA), a non-profit organization that promotes, educates, and provides standards of practice, ethics, and safety in EQT [35]. Since 1997, EAGALA has hosted an annual conference for mental health professionals to share their observations and experiences with EQT, exchange ideas, and network. ED specialists who practice EQT also frequently seek certification from EAGALA to offer formalized Equine Assisted Psychotherapy [35].

Due to the frequent use of EQT with ED inpatients and in light of the paucity of research into its efficacy, this investigation has been designed to study the efficacy of a structured EQT program with ED inpatients using well-validated and reliable outcome measures. In particular, this investigation targets previous observations that EQT may positively impact the specific ED and common co-occurring symptoms of drive for thinness, impaired self-efficacy, interpersonal distrust, impulse dysregulation, depressed mood, and anxiety in ED patients [37-39]. This is an initial attempt to move EQT research into stage 2 testing [34].

\section{Method}

\subsection{Participants}

The sample included 72 female inpatients treated at an inpatient ED facility between December 2005 and April 2008. The sample was randomly selected from the population of patients admitted during the specified time frame. All patients completed their prescribed stay in treatment, i.e., did not drop out of treatment prematurely, and thus completed a full course of standardized, manualized inpatient treatment for EDs.

Participant admission age ranged from $18-49$ years $(M=26, S D=7.5)$. Thirty-two percent of the 
participants had a diagnosis of anorexia nervosa, 33\% bulimia nervosa, and 35\% ED not otherwise specified. At admission, $66 \%$ of the participants had never been married, $19 \%$ were married, and $4 \%$ widowed. The majority, $42 \%$, had some college education; $34 \%$ had a bachelors degree or some postgraduate education; $24 \%$ had a high school diploma; and $1 \%$ had less than a high school education. In terms of racial/ethnic self-identification, $92 \%$ of the patients self-identified as White; one patient each self-identified as African-American, Asian-American, and Native-American; and three self-identified as multi-racial.

\subsection{Materials}

We select assessments that measure the constructs on which EQT has been reported previously to have a positive therapeutic influence. We use well-validated and reliable instruments to measure EQT outcomes: the Eating Disorder Inventory-2 [41], Beck Depression Inventory-II [42], and Beck Anxiety Inventory [43]. The EDI-2 is a self-report measure of symptoms commonly associated with EDs. Four EDI-2 subscales related to the hypothesized benefits of EQT are utilized in this study: drive for thinness (DT); ineffectiveness (I) as a measure of impaired self-efficacy; interpersonal distrust (ID); and impulse regulation (IR). The BDI-II is a self-report instrument measuring the severity of depressive symptoms. The BAI is a self-report instrument measuring the severity of generalized anxiety symptoms.

Participation in EQT is measured by the total number of minutes spent in any EQT activity during treatment, Total Equine Minutes (TEM). Using TEM permits not only a comparison of patients who receive standardized treatment alone to those who received standardized treatment plus EQT, but also a measurement of dosage effects, i.e., the efficacy of lesser or greater amounts of EQT in addition to standardized treatment.

Because treatment at the inpatient facility from which the sample is drawn is standardized and manualized according to ED and co-occurring diagnoses, length of stay in treatment (LOS) is chosen as a suitable measure of the amount of standardized treatment received by participants in this study. LOS is the logical analogue of TEM; TEM measures time spent in EQT, while LOS measures time spent receiving standardized treatment.

\subsection{Standard Treatment}

All patients participate in a standardized, manualized regimen of inpatient treatment for EDs offering cognitive-behavioral and dialectical behavioral therapy, family systems therapy, nutrition counseling and education, and medical and psychotropic medication management, using both individual and group formats. At the time of the study, the facilitys standardized treatment protocols are designed to follow the American Psychiatric Associations Practice Guideline for the Treatment of Patients with Eating Disorders, Third Edition [44]. A detailed manual of the standardized treatment components is available from the author. All staff who provide components of the standardized treatment are licensed healthcare professionals, including board-certified psychiatrists and psychiatric nurse practitioners, licensed doctoral level psychologists, licensed masters level counselors, and licensed practical and registered nurses. All staff are trained in the facilitys manualized approach to ED treatment during a two-week initial orientation followed by a three-month observation by a supervisor or mentor in the same profession. All patients in the study are randomly assigned to their treatment providers. 


\subsection{EQT Interventions}

Experienced equine therapists are trained and certified in Equine Assisted Psychotherapy by EAGALA. All patients are randomly assigned to their equine therapist. The equine therapists utilize a standardized EQT protocol with several components, as follows.

Firstly, equine therapists select horses for characteristics making them suitable to patient contact.

Secondly, horses receive initial and regularly repeated training for successful and safe participation in EQT.

Thirdly, patients are paired with specific horses based on an assessment of patient riding needs and experiences.

Fourthly, patients are allowed to participate in EQT only if approved by a licensed physician. Once approved, all patients participate in a minimum of one EQT session per week, with additional sessions scheduled at the option of each patients treatment team when the needs listed above as amenable to EQT are identified for the patient.

Finally, during EQT sessions, all patients participate in a range of EQT activities, including ground activities such as grooming, harness driving, lariat activity, and longe line training; mounted activities such as trail rides, arena riding at the walk, rising trot, and canter; and manualized experiential exercises akin to psychodrama with one or more horses. Equine therapists emphasize specific activities for each patient based on the patients therapeutic needs identified by the treatment team.

\subsection{Statistical Treatment}

Because subjects receive variable amounts of EQT, rather than a basic treatment outcome design comparing a non-EQT group to an EQT-group, we utilize an outcome design capable of capturing the effects of differential doses of EQT [45, 46]. Thus, to compare the efficacy of standardized treatment to that of standardized treatment plus EQT in ED patients, we run six linear regression models-one model for each outcome measure. In each model, the dependent variable is the discharge score of the outcome measure. TEM is the independent variable, with the admission score on the same outcome measure plus LOS entered into the equation as control variables.

We include in the equations the admission score for each outcome measure to statistically control for the severity of each symptom at admission, so that any relationship between TEM and a lower discharge score cannot be attributed merely to a baseline condition of less severity for that symptom. We include LOS in the analyses to control for the amount of standardized treatment experienced by all subjects, allowing any additional effects of EQT to be isolated from the standardized treatment. Because the regression models are designed with these control variables, any relationship found between TEM and a lower discharge score on the six outcome measures can be attributed to the amount of EQT itself and not to a greater or lesser amount of standardized treatment received by the patients.

The following regression equations are computed:

1) EDI-2 DT discharge score $=$ TEM + EDI-2 DT admission score + LOS

2) EDI-2 I discharge score = TEM + EDI-2 I admission score + LOS

3) $\mathrm{EDI}-2$ ID discharge score $=$ TEM + EDI-2 ID admission score + LOS

4) EDI-2 IR discharge score $=$ TEM + EDI-2 IR admission score + LOS

5) BDI-II discharge score $=$ TEM + BDI-II admission score + LOS

6) $\mathrm{BAI}$ discharge score $=\mathrm{TEM}+\mathrm{BAI}$ admission score $+\mathrm{LOS}$ 
Table 1. Regression Results for the Efficacy of EQT in Reducing Six ED Symptoms

\begin{tabular}{|c|c|c|c|c|c|c|c|c|}
\hline \multirow{2}{*}{ Model } & \multirow{2}{*}{$\mathrm{F}$} & \multirow{2}{*}{$\mathrm{p}$} & \multicolumn{6}{|c|}{ EQT (Total Equine Minutes) } \\
\cline { 5 - 9 } & & & $\mathrm{t}$ & $\mathrm{p}$ & Beta & \multicolumn{2}{|c|}{ SE Beta } & 95\% Confidence Interval for B \\
\hline EDI-2 Drive for Thinness & 10.084 & $<.001$ & -2.042 & .04 & -.005 & .003 & -.011 & -.0001 \\
\hline EDI-2 Ineffectiveness & 12.497 & $<.001$ & -2.827 & .006 & -.008 & .003 & -.014 & -.002 \\
\hline EDI-2 Interpersonal Distrust & 8.061 & $<.001$ & -1.969 & .05 & -.003 & .002 & -0007 & .0000 \\
\hline EDI-2 Impulse Regulation & 20.476 & $<.001$ & -3.332 & 0.001 & -.005 & .002 & -.009 & -.002 \\
\hline BDI-II & 9.400 & $<.001$ & -2.726 & .008 & -.015 & .005 & -.025 & -.004 \\
\hline BAI & 11.524 & $<.001$ & -2.539 & .01 & -.012 & .005 & -.022 & -.003 \\
\hline
\end{tabular}

\section{Results}

All six models are highly statistically significant; see Table 1 . More importantly, TEM is significant at an alpha of .05 in models 1 and 3, and at or greater than .01 in models 2, 4, 5, and 6. These results suggest that there is a significant relationship between the number of minutes spent in EQT and patients discharge scores on drive for thinness, ineffectiveness, interpersonal distrust, impulse regulation, depression, and anxiety. As predicted, in all models there is an inverse relationship between TEM and discharge scores, indicating that as the dosage of EQT increases, discharge scores decrease.

Beta coefficients from the models indicate that for each one hour increase in EQT-the equivalent of one EQT session-the EDI-2 drive for thinness score decrease by .3, ineffectiveness by .48, interpersonal distrust by .18, impulse regulation by .3, BDI-II depression score by .9 , and BAI anxiety score by .72 .

\section{Discussion}

The present study compares a standard inpatient treatment for EDs to standard treatment plus EQT. We predicte that the addition of EQT will reduce ED patients drive for thinness, impaired self-efficacy, interpersonal distrust, impulse dysregulation, depressed mood, and anxiety. All six hypotheses are confirmed. For ED inpatients, adding EQT appears related to a decreased drive for thinness and improved self-efficacy, interpersonal trust, impulse regulation, depression, and generalized anxiety.

For each symptom, Beta coefficients from the regression equations suggest that the amount of symptom improvement gained from even four one-hour EQT sessions may represent clinically meaningful additions to standard treatment, ranging anywhere from a one to a four point reduction on the various outcome measures. This association between EQT and improvements in key ED and co-occurring symptoms may suggest a need for further research into EQT as an adjunctive treatment modality for EDs in addition to standard treatment protocols.

EQT specialists suggest that equine activities help patients to develop a more realistic view of themselves through awareness of their size in relation to the horse. The relationship between EQT and reduction in drive for thinness lends support to this clinical observation. EQT specialists also suggest that the learning and mastery of horsemanship, a new skill for most patients, enhances patients confidence in their ability to tackle new projects, such as those involved in recovery, and that learning to communicate and achieve harmony with a large animal promotes the experience of self-efficacy instead of helplessness. The relationship between EQT and reduction in feelings of ineffectiveness lends support to this clinical observation as well. EQT specialists suggest that, by learning to trust an animal such as a horse during EQT, patients develop or renew a capacity for trust, which they then generalize to human beings. One observational study specifically finds parallels between human-horse bonding and an improved therapeutic 
alliance between clients and their treatment providers [16]. The relationship we find between EQT and reduction in interpersonal distrust lends support to this observation.

Prior research indicates that animal-assisted therapies may reduce patient agitation and aggressiveness and increase cooperativeness and behavioral control, because the need to communicate with an animal calmly and non-reactively promotes the skills of emotional awareness, emotion and impulse regulation, and self-control [11, 12]. The relationship we find between EQT and reduction in impulse dysregulation suggests that this effect of animal-assisted therapy may also occur for ED patients engaged in EQT.

Prior research further suggests that animal-assisted therapies may reduce depressive symptoms for several reasons [2, 4]: patients rediscover joy through encounters with the outdoors and an appreciation for the uniqueness of the animal they are working with; patients reduce their absorbing, depressive ruminations because they direct their attention outwardly toward safe and caring interactions with the therapy animal; patients rediscover spontaneity and the ability for healthy recreation through the playful aspects of animal-assisted therapy, thus reducing the experience of anhedonia. The relationship we find between EQT and a reduction in depressive symptoms suggests that these effects of animal-assisted therapy may also occur for ED patients engaged in EQT.

Finally, previous research reliably indicates that human-animal contact reduces physiological anxiety levels and thus patients experiences of generalized anxiety (e.g., [6]). The relationship we find between EQT and reduction in anxiety suggests that this effect may also occur for ED patients engaged in EQT.

This study has several limitations. First, all patients voluntarily seek admission, or are persuaded by family members to admit, to an inpatient treatment facility specializing in EDs. Although the samples sociodemographic characteristics appear similar to the sociodemo graphics of most inpatient ED populations, various issues, including their treatment-seeking status, may hamper the generalizability of this study. Second, due to limitations in sample size, the study combines patients with the several ED diagnoses into one group. Future research might attempt to establish a clearer understanding of whether ED patients with the several diagnoses and other distinguishing features may differentially benefit from EQT. Third, the study considers all EQT activities equivalently. Future research might attempt to isolate particular modes of EQT to determine which has the greatest efficacy and cost effectiveness for specific symptoms. Fourth, all patients begin EQT immediately upon approval by a physician, whereas future research might attempt to establish at what point in treatment the introduction of EQT is most beneficial in relation to specific symptoms. Fifth, the outcome measures are obtained at time of discharge from inpatient treatment. Longer-term outcome measurements should follow in future research to assess the longevity of the symptom reductions. Sixth, the standard treatment received by all patients includes multiple components. Although most inpatient facilities in the United States attempt to follow the American Psychiatric Associations Practice Guideline for the Treatment of Patients with Eating Disorders, Third Edition, it is known in the field that substantial variability exists across facilities. In a sense, there is no truly standard inpatient treatment for EDs. A multi-site study with more formal controls on standard treatment protocols and staff training will be optimal, but costly and difficult to operationalize. With this present limitation, the current study can at most be said to establish the possibility that EQT may further reduce several key ED symptoms when applied in addition to one facilitys standardized, manualized treatment protocols. Nevertheless, it is worth underscoring that this finding of treatment efficacy for EQT, however limited it may be, represents new and suggestive information about EQT with ED patients. Since EQT is a therapeutic modality that is already widely used in modern ED treatment, this finding may be valuable as an incremental step toward more extensive research attention to the efficacy of EQT. Finally, although this study suggests initial efficacy for EQT, it is a costly intervention due to the need to acquire and maintain horses, riding facilities, and equipment. This significant factor, as well as other issues, will ultimately need to be addressed within treatment effectiveness research. 


\section{Acknowledgments}

All of us would like to thank the equine therapy mentors, especially Sharon Simpson and Cheryl Musick.

\section{References}

[1] S. Elliott, J. Funderburk, and J. Holland, "The impact of the stirrup some fun therapeutic horseback riding program: A qualitative investigation," American Journal of Recreation Therapy, vol. 7, no. 2, pp. 19-28, 2008.

[2] G. Francis, J. T. Turner, and S. B. Johnson, "Domestic animal visitation as therapy with adult home residents," International Journal of Nursing Studies, vol. 22, no. 3, pp. 201-206, 1985.

[3] M. L. Morrison, "Health benefits of animal-assisted interventions," Complementary Health Practice Review, vol. 12, no. 1, pp. 51-62, 2007.

[4] M. A. Souter and M. D. Miller, "Do animal-assisted activities effectively treat depression? a metaanalysis," Anthrozoos: A Multidisciplinary Journal of The Interactions of People \& Animals, vol. 20, no. 2, pp. 167-180, 2007.

[5] K. S. Trotter, C. K. Chandler, D. Goodwin-Bond, and J. Casey, "A comparative study of the efficacy of group equine assisted counseling with at-risk children and adolescents," Journal of Creativity in Mental Health, vol. 3, no. 3, pp. 254-284, 2008.

[6] S. B. Barker and K. S. Dawson, "The effects of animal-assisted therapy on anxiety ratings of hospitalized psychiatric patients," Psychiatric Services, vol. 49, no. 6, pp. 797-801, 1998.

[7] S. B. Barker, A. K. Pandurangi, and A. M. Best, "Effects of animal-assisted therapy on patients' anxiety, fear, and depression before ect," The journal of ECT, vol. 19, no. 1, pp. 38-44, 2003.

[8] H. Greaves, "Womens metamorphosis with horses," G. Pony Boy, Of Women and Horses, pp. 9-13, 2000.

[9] H. R. Glazer, M. D. Clark, and D. S. Stein, "The impact of hippotherapy on grieving children," Journal of Hospice \& Palliative Nursing, vol. 6, no. 3, pp. 171-175, 2004.

[10] A. H. Katcher and G. G. Wilkins, "The use of animal assisted therapy and education with attentiondeficit hyperactive and conduct disorders," Interactions, vol. 12, no. 4, pp. 1-5, 1994.

[11] G. P. Mallon, "Utilization of animals as therapeutic adjuncts with children and youth: A review of the literature," in Child and Youth Care Forum, vol. 21, pp. 53-67, Springer, 1992.

[12] A. McCormick and M. McCormick, "Horse sense and the human heart: What horses can teach us about trust, bonding, creativity, and spirituality," Deerfield, FL.: Health Communications Inc, 1997.

[13] J. C. Arnold, "Therapy dogs and the dissociative patient: preliminary observations," Dissociation, vol. 8, pp. 247-252, 1995.

[14] N. Parish-Plass, "Animal-assisted therapy with children suffering from insecure attachment due to abuse and neglect: a method to lower the risk of intergenerational transmission of abuse?", Clinical Child Psychology and Psychiatry, vol. 13, no. 1, pp. 7-30, 2008.

[15] P. N. Schultz, G. Remick-Barlow, and L. Robbins, "Equine-assisted psychotherapy: A mental health promotion/intervention modality for children who have experienced intra-family violence," Health \& Social Care in the Community, vol. 15, no. 3, pp. 265-271, 2007.

[16] J. Yorke, C. Adams, and N. Coady, "Therapeutic value of equinehuman bonding in recovery from trauma," Anthrozoos: A Multidisciplinary Journal of The Interactions of People \& Animals, vol. 21, no. 1, pp. 17-30, 2008.

[17] K. Batson, B. W. McCabe, and M. M. Baun, "The effect of a therapy dog on socialization and 
physiologic indicators of stress in persons diagnosed with alzheimers disease," Animals, Health and Quality of Life, 1995.

[18] S. L. Filan and R. H. Llewellyn-Jones, "Animal-assisted therapy for dementia: a review of the literature," International Psychogeriatrics, vol. 18, no. 04, pp. 597-611, 2006.

[19] C. L. Fritz, T. B. Farver, P. H. Kass, and L. A. Hart, "Association with companion animals and the expression of noncognitive symptoms in alzheimer's patients," The Journal of nervous and mental disease, vol. 183, no. 7, pp. 459-463, 1995.

[20] J. Tribet, M. Boucharlat, M. Myslinski, et al., "[animal-assisted therapy for people suffering from severe dementia]," L'Encephale, vol. 34, no. 2, pp. 183-186, 2008.

[21] M. M. Bass, C. A. Duchowny, and M. M. Llabre, "The effect of therapeutic horseback riding on social functioning in children with autism," Journal of Autism and Developmental Disorders, vol. 39, no. 9, pp. 1261-1267, 2009.

[22] C. Campbell and A. Katcher, "Animal assisted therapy dogs for autistic children: Quantitative and qualitative results," in Sixth International Conference on Human-Animal Interactions. Montreal, 1992.

[23] L. Kaiser, K. A. Smith, C. R. Heleski, and L. J. Spence, "Effects of a therapeutic riding program on at-risk and special education children," Journal of the American Veterinary Medical Association, vol. 228, no. 1, pp. 46-52, 2006.

[24] B. L. Macauley and K. M. Gutierrez, "The effectiveness of hippotherapy for children with languagelearning disabilities," Communication Disorders Quarterly, vol. 25, no. 4, pp. 205-217, 2004.

[25] F. Martin and J. Farnum, "Animal-assisted therapy for children with pervasive developmental disorders," Western Journal of Nursing Research, vol. 24, no. 6, pp. 657-670, 2002.

[26] A. M. Beck, L. Seraydarian, and G. F. Hunter, "Use of animals in the rehabilitation of psychiatric inpatients," Psychological Reports, vol. 58, no. 1, pp. 63-66, 1986.

[27] B. Berget, Ø. Ekeberg, and B. O. Braastad, "Animal-assisted therapy with farm animals for persons with psychiatric disorders: effects on self-efficacy, coping ability and quality of life, a randomized controlled trial," Clinical practice and epidemiology in mental health, vol. 4, no. 1, p. 9, 2008.

[28] C. A. Ewing, P. M. MacDonald, M. Taylor, and M. J. Bowers, "Equine-facilitated learning for youths with severe emotional disorders: A quantitative and qualitative study," in Child and Youth Care Forum, vol. 36, pp. 59-72, Springer, 2007.

[29] J. Hundley, "Pet project. the use of pet facilitated therapy among the chronically mentally ill.," Journal of psychosocial nursing and mental health services, vol. 29, no. 6, pp. 23-26, 1991.

[30] Z. Kovács, R. Kis, S. Rózsa, and L. Rózsa, "Animal-assisted therapy for middle-aged schizophrenic patients living in a social institution. a pilot study.," Clinical Rehabilitation, vol. 18, no. 5, 2004.

[31] I. Nathans-Barel, P. Feldman, B. Berger, I. Modai, and H. Silver, "Animal-assisted therapy ameliorates anhedonia in schizophrenia patients," Psychotherapy and Psychosomatics, vol. 74, no. 1, pp. 31-35, 2004.

[32] B. T. Klontz, A. Bivens, D. Leinart, and T. Klontz, "The effectiveness of equine-assisted experiencial therapy: Results of an open clinical trial," Society and Animals, vol. 15, no. 3, p. 257, 2007.

[33] J. Nimer and B. Lundahl, "Animal-assisted therapy: A meta-analysis," Anthrozoos: A Multidisciplinary Journal of the Interactions of People \& Animals, vol. 20, no. 3, pp. 225-238, 2007.

[34] B. J. Rounsaville, K. M. Carroll, and L. S. Onken, "A stage model of behavioral therapies research: Getting started and moving on from stage i," Clinical Psychology: Science and Practice, vol. 8, no. 2, pp. 133-142, 2001.

[35] EAGALA, "Fundamentals of eagala model practice.," 2006.

[36] P. Mandrell, Introduction to equine-assisted psychotherapy. Xulon Press, 2006.

[37] A. L. Bizub, A. Joy, and L. Davidson, "its like being in another world: Demonstrating the benefits of 
therapeutic horseback riding for individuals with psychiatric disability," Psychiatric Rehabilitation Journal, vol. 26, no. 4, pp. 377-384, 2003.

[38] J. E. Christian, "All creatures great and small utilizing equine-assisted therapy to treat eating disorders," Journal of Psychology and Christianity, vol. 24, no. 1, pp. 65-67, 2005.

[39] E. J. CUMELLA, "Questions \& answers," Eating Disorders, vol. 11, no. 2, pp. 143-147, 2003.

[40] M. Zugich, T. Klontz, and D. Leinart, "The miracle of equine therapy," Counselor Magazine, vol. 3, no. 6, pp. 22-27, 2002.

[41] D. M. Garner, Eating disorder inventory-2: Professional manual. psychological assessment resources Odessa, FL, 1991.

[42] A. Beck, R. Steer, and G. Brown, "Beck depression inventory. 2nd edn, the psychological corporation," San Antonio, 1996.

[43] A. Beck and R. Steer, "Beck anxiety inventory manual. san antonio, tx: The psychological corporation," 1993.

[44] J. Yager, M. J. Devlin, K. A. Halmi, D. B. Herzog, J. E. Mitchell, and e. a. Powers, P., "Practice guideline for eating disorders (3rd ed.)," American Journal of Psychiatry., 2006.

[45] A. E. Bergin and S. L. E. Garfield, Handbook of psychotherapy and behavior change. John Wiley \& Sons, 1994.

[46] L. Siqueland, A. Frank, D. Gastfriend, L. Muenz, P. Crits-Christoph, J. Chittams, M. Thase, D. Mercer, and J. Blaine, "The protocol deviation patient: characterization and implications for clinical trials research," Psychotherapy Research, vol. 8, no. 3, pp. 287-306, 1998. 